\title{
Diagnostic utility of IMP3 in segregating metastatic melanoma from benign nevi in lymph nodes
}

\author{
Mark J Mentrikoski ${ }^{1}$, Linglei Ma ${ }^{2}$, Jennifer G Pryor ${ }^{1}$, Loralee A McMahon ${ }^{1}$, Qi Yang ${ }^{1}$, \\ Betsy O Spaulding ${ }^{3}$, Glynis A Scott ${ }^{4}$, Hanlin L Wang ${ }^{5}$ and Haodong $\mathrm{Xu}^{1}$ \\ ${ }^{1}$ Department of Pathology and Laboratory Medicine, University of Rochester Medical Center, Rochester, \\ NY, USA; ${ }^{2}$ Department of Pathology and Dermatology, University of Michigan Medical Center, Ann Arbor, \\ MI, USA; ${ }^{3}$ Dako North America, Carpinteria, CA, USA $;{ }^{4}$ Department of Dermatology, University of Rochester \\ Medical Center, Rochester, NY, USA and ${ }^{5}$ Department of Pathology and Laboratory Medicine, \\ Cedars-Sinai Medical Center, Los Angeles, CA, USA
}

\begin{abstract}
Depending on the Breslow depth of the primary melanoma, sentinel lymph node biopsy is considered as standard of care for the staging of cutaneous melanoma, and is one of the most important prognostic factors. The histologic analysis of these specimens becomes difficult to interpret when benign intranodal nevic cells mimic metastases. Insulin-like growth factor-II messenger RNA (mRNA)-binding protein-3 (IMP3), also known as $\mathrm{K}$ homology domain-containing protein overexpressed in cancer or L523S, is a member of the insulin-like growth factor-II mRNA-binding protein family and has been shown to have diagnostic utility in distinguishing cutaneous melanoma from benign nevi. In this study, 43 sentinel lymph node biopsy specimens, including 13 with benign intranodal nevi and $\mathbf{3 0}$ with metastatic melanoma (two cases containing both benign nevi and metastatic melanoma), from 41 patients were immunohistochemically analyzed with a monoclonal antibody against IMP3. None of the benign intranodal nevi expressed IMP3, whereas 21 out of $30(70 \%)$ of the lymph nodes containing metastatic melanoma did. It seems that IMP3 is helpful in distinguishing benign intranodal nevi from metastatic melanoma in sentinel lymph node biopsy specimens, and could be a valuable diagnostic adjunct in sentinel lymph node biopsy assessment in which questions arise as to the malignancy of the melanocytes present.

Modern Pathology (2009) 22, 1582-1587; doi:10.1038/modpathol.2009.128; published online 4 September 2009
\end{abstract}

Keywords: melanoma; metastatic melanoma; nodal nevus; sentinel lymph node; IMP3

For patients with a diagnosis of cutaneous melanoma, sentinel lymph node biopsy is largely considered as standard of care. First described in melanoma by Morton et al in 1992, ${ }^{1}$ sentinel lymph node biopsy with microscopic evaluation ranks as one of the most important primary disease prognostic factors. ${ }^{2}$ In the case of a positive sentinel lymph node, patients often undergo further interventions including completion lymph node dissection and various chemotherapeutic regimens; as a positive

Correspondence: Dr H Xu, Department of Pathology and Laboratory Medicine, University of Rochester Medical Center, 601 Elmwood Avenue, Box 626, Rochester, New York, 14642, USA. E-mail: Haodong_Xu@urmc.rochester.edu

Received 4 June 2009; revised and accepted 27 July 2009; published online 4 September 2009 sentinel lymph node signifies a $20 \%$ chance that other nodes harbor metastases and a drop in diseasespecific 5 year survival rate from 90 to $72 \%{ }^{2,3}$ Thus, because sentinel lymph node biopsy provides important predictive value and treatment guidance for melanoma, it is critical that the histological assessment of sentinel lymph node biopsy specimens is thorough and accurate.

To aid in the identification of metastatic foci, immunohistochemical stains that rely on melanocytic features such as Melan-A/MART-1 and S-100 are commonly used. These stains become difficult to interpret when benign nevic cells, so-called nodal nevi, mimic metastases within sentinel lymph node specimens. Although it is unknown how these nevus cells arise in lymph nodes, their presence has been known since 1931. ${ }^{4}$ The frequency in 
which nodal nevi are found varies widely and is estimated to occur in as frequent as $8 \%$ of nonmelanoma specimens. ${ }^{5-7}$ Not surprisingly, this estimate is thought to be increased in melanoma cases ${ }^{8,9}$ and sentinel lymph node biopsy specimens specifically. ${ }^{8,10,11}$ When nodal nevi are encountered, they are typically located within the fibrous capsule or trabeculae of the lymph node; however, actual intraparenchymal benign nevic cells have occasionally been reported..$^{5,7,12-14}$

Recently, an immunohistochemical marker, insulin-like growth factor-II messenger RNA (mRNA)binding protein-3 (IMP3), has been reported to differentiate between melanomas and benign nevi. ${ }^{15}$ IMP3, also known as K homology domain-containing protein overexpressed in cancer or L523S, promotes tumor cell proliferation by enhancing insulin-like growth factor-II protein expression. ${ }^{16}$ It is a 580 amino-acid protein with $4 \mathrm{~K}$ homology domains and 2 RNA recognition motifs and is encoded by a gene on chromosome $7 \mathrm{p} 11.5 .{ }^{17} \mathrm{IMP} 3$ is normally expressed in lymph node germinal centers, focal intestinal mucosa, placenta, ovary, testis, and brain. ${ }^{18-20}$ Aside from being overexpressed in melanoma, IMP3 has also been identified in a number of malignancies including pancreatic cancer, renal cell carcinoma, germ cell neoplasms, and lung carcinomas. ${ }^{19-24}$

As IMP3 has diagnostic utility in discriminating cutaneous melanoma from benign nevi, we hypothesize that expression of IMP3 can also be used to differentiate metastatic melanoma from benign intranodal nevi in sentinel lymph node biopsy specimens. To test this hypothesis, we explored IMP3 expression in sentinel lymph node biopsy specimens with known metastatic melanoma and benign intranodal nevi by immunohistochemistry.

\section{Materials and methods}

\section{Case selection}

This study was conducted in accordance with a protocol approved by the institutional review board. A total of 43 specimens were retrieved from the surgical pathology archives at University of Rochester, University of Michigan, and Cedars-Sinai Medical Centers. Of the 43 total specimens, 13 sentinel lymph node biopsies contained benign nodal nevi from 12 subjects. The benign nevi were located both in the capsule $(n=11)$ and trabeculae $(n=2)$. In addition, 25 sentinel and 5 non-sentinel lymph nodes with confirmed metastatic melanoma from 29 subjects were retrieved. Two cases contained both metastatic melanoma and benign nodal nevi. Hematoxylin and eosin (H\&E) and MelanA/MART-1-stained slides were examined for each case. Nevic cells were highlighted on the MelanA-stained slides, and a diagnosis of melanoma was based on cytological atypia, location of the atypical cells, and comparison with the primary melanomas.

\section{Immunohistochemical Analysis}

Immunohistochemical studies were performed on $5-\mu \mathrm{m}$ formalin-fixed, paraffin-embedded tissue sections using a mouse monoclonal antibody against IMP3 (clone 69.1; Dako North America, Carpinteria, CA, USA). Tissue sections were deparaffinized according to established procedures and quenched with $3 \% \mathrm{H}_{2} \mathrm{O}_{2}$ for $6 \mathrm{~min}$. Sections were washed in running water and Tris-buffered saline consisting of $50 \mathrm{mmol} / \mathrm{l}$ Tris-HCl (pH 7.6), $150 \mathrm{mmol} / \mathrm{l} \mathrm{NaCl}$, and $0.05 \%$ Tween 20 . Antigen retrieval was performed using preheated $\left(95-99^{\circ} \mathrm{C}\right)$ target retrieval solution, modified citrate buffer, $\mathrm{pH} 6.1$ (Dako) in a Black and Decker steamer (Model HS800; Shelton, CT, USA) for $30 \mathrm{~min}$, followed by a 15-min cool down period. Slides were rinsed with Tris-buffered saline for $5 \mathrm{~min}$ and mounted in the Dako Autostainer. Sections were incubated with the monoclonal mouse anti-IMP3 antibody (dilution 1:100) at room temperature for 40-60 min. The sections were then incubated for 30 min with EnVision + System horseradish peroxidase-labeled polymer conjugated to goat anti-mouse (Dako). Staining was developed with 3,3'-diamino-benzidine tetrahydrochloride + substrate-chromogen (Dako) for $10 \mathrm{~min}$. Slides were rinsed in running distilled water, counterstained with modified Mayer's hematoxylin, and blued in $0.3 \%$ ammonia water followed by a tap water rinse. Slides were mounted using an aqueous medium and viewed under a light microscope. Care was taken so that the cells positive for Melan-A were the same cells examined when evaluating for IMP3 expression. IMP3 is known to be expressed in lymph node germinal center cells, which serves as internal positive controls. Cytoplasmic staining was considered as positive for IMP3 expression. The percentage of tumor cells with cytoplasmic staining was recorded, with $>10 \%$ of cells staining considered positive. The intensity of cytoplasmic staining was recorded as weak, moderate, or strong.

\section{Results}

\section{IMP3 Immunohistochemical Staining}

The results of immunohistochemical studies of IMP3 expression in 43 sentinel lymph node biopsy specimens are summarized in Table 1. Germinal

Table 1 IMP3 and Melan-A staining in benign nodal nevi and metastatic melanoma

\begin{tabular}{lcr}
\hline & IMP3+ & Melan-A+ \\
\hline Benign capsular nevi $(n=11)$ & $0(0 \%)$ & $11(100 \%)$ \\
Benign trabecular nevi $(n=2)$ & $0(0 \%)$ & $2(100 \%)$ \\
Melanoma $(n=30)$ & $21(70 \%)$ & $30(100 \%)$ \\
\hline
\end{tabular}

Note: + , positive. 
centers were highlighted in all lymph nodes and served as internal positive controls (Figure 2b). None of the lymph nodes containing benign nodal nevi expressed IMP3 (Figure 1b), whereas all were positive for Melan-A/MART-1 (Figure 1c). Care was taken so that cells staining positive for Melan-A were the same cells examined when evaluating IMP3 expression (Figure $1 \mathrm{~b}$ and c). Twenty one out of $30(70 \%)$ lymph nodes containing metastatic melanoma were immunohistochemically positive for IMP3 with $>10 \%$ of tumor cells stained (Figure 2b) and for Melan-A/MART-1 (Figure 2c). Immunostaining intensity ranged from weak to strong. The overall sensitivity was $70 \%$ and specificity $100 \%$.

\section{Discussion}

Owing to the prognostic value and therapeuticdirecting information afforded by the sentinel lymph node biopsy procedure in the evaluation of a patient with primary cutaneous melanoma, proper specimen analysis is imperative. Our earlier study showed that IMP3 is likely a novel progression marker in melanoma, and that it has the ability to differentiate benign melanocytic nevi from malignant melanoma. ${ }^{15}$ This study reaffirms those earlier findings and shows that IMP3 has diagnostic utility in distinguishing metastatic melanoma from benign nodal nevi in sentinel lymph node biopsy specimens.
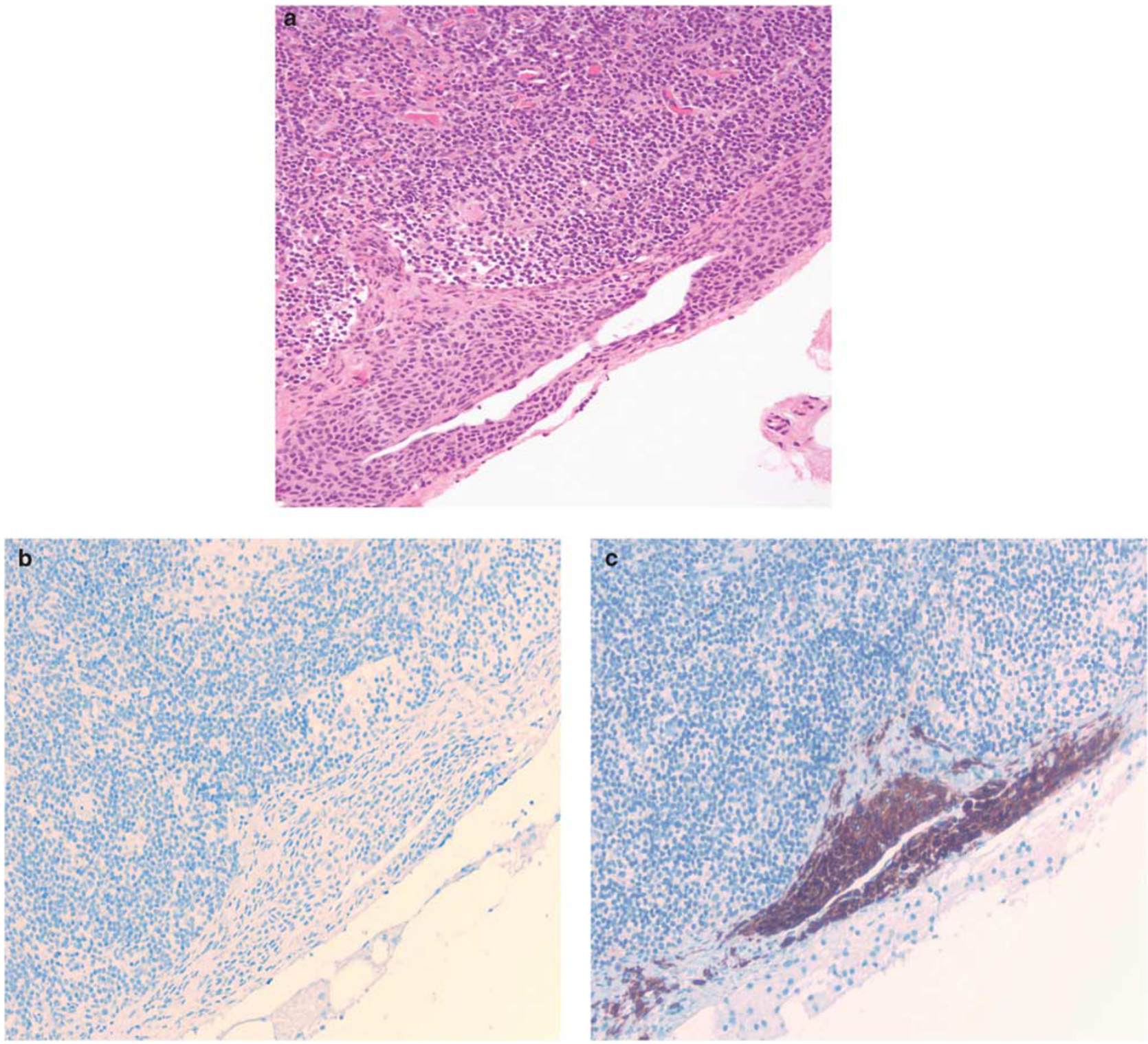

Figure 1 (a) Histology of sentinel lymph node with intracapsular nevic cells (original magnification $\times 200$ ). (b, c) Intracapsular nevic cells showing complete lack of IMP3 staining and strong Melan-A positivity (original magnification $\times 200$ ). 

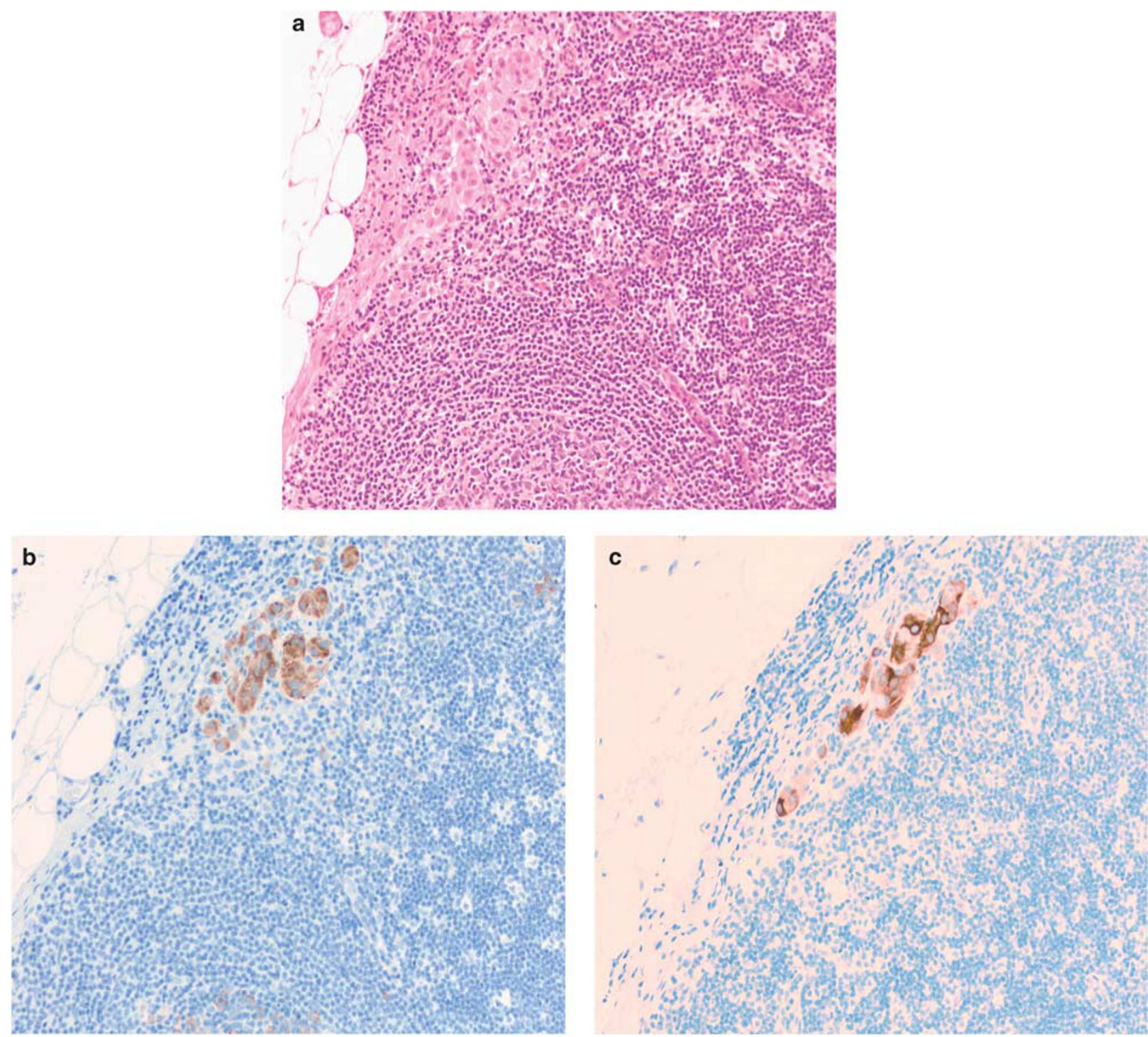

Figure 2 (a) Histology of sentinel lymph node with subcapsular metastatic melanoma (original magnification $\times 200$ ). (b) Melanoma showing strong cytoplasmic IMP3 staining in $>90 \%$ of cells and moderate IMP3 positivity lymphocytes in germinal center (original magnification $\times 200$ ). (c) Melanoma exhibiting strong cytoplasmic Melan-A staining (original magnification $\times 200$ ).

In general, when present in large, cohesive cell clusters within the lymph node parenchyma, metastatic melanoma can be easily identified on H\&E sections. Similarly, benign nodal nevi that present as groups of small, bland, intracapsular cells can often be correctly diagnosed without the assistance of immunohistochemical markers. However, because micrometastases of melanoma occur frequently and are likely missed on H\&E sections, many institutions use various immunohistochemical stains to aid in sentinel lymph node biopsy examination. Problems arise when small foci of positive cells are located in the trabueculae of the lymph node with inconspicuous cytological atypia.

The immunohistochemical stains commonly used in the workup of questionable sentinel lymph node biopsy specimens include S-100, Melan-A/MART-1, and/or HMB-45. S-100 is the most sensitive for detecting metastatic melanoma, though it is rather non-specific and stains benign nodal melanocytes, dendritic cells, melanophages, adipose tissue, and nerves. Melan-A/MART-1, an immunohistochemical stain with increased melanocyte specificity, has also been used to screen sentinel lymph node biopsies, but it identifies both benign intranodal nevi and metastatic melanomas. Even though Melan-A is an extremely specific melanocyte marker, it does not provide distinction between melanoma cells and nevic cells. HMB-45, such as Melan-A, also has increased specificity for melanocytes compared with S-100. In addition, HMB-45 appears to be more helpful in distinguishing nodal nevi than S-100 and Melan-A because it is typically negative in benign melanocytes, ${ }^{25}$ although it has 
been shown to be positive in up to $16 \%$ of sentinel lymph node biopsy benign nevic cell rests. ${ }^{26}$ HMB45 also has a tendency to stain melanophages, which can mimic micrometastases. However, much like IMP3, the utility of HMB-45 can be limited because it is overall less sensitive in detecting metastatic melanoma, creating false negative results. ${ }^{27-29}$

With each of the melanocytic immunohistochemical stains having its own drawbacks, there has been no consensus as to which is the most appropriate marker to use when questionable cases arise. Currently, a combination of the above stains is used for screening and diagnostic purposes. When deposits within the lymph nodes are large, diagnosis of metastasis versus intranodal nevi can be made based on location and cytological pleomorphism of the cells on $\mathrm{H} \& \mathrm{E}$ stain alone. When small foci of S-100 or Melan-A positive cells occur within the lymph node trabeculae or parenchyma, so-called micrometastases, the outright diagnosis of metastatic melanoma can be more difficult. Our study shows that IMP3 expression can serve as a diagnostic adjunct in distinguishing benign intranodal nevi from metastatic melanomas when used together with other melanocytic markers. However, it should be noted that the cases used in this study contained specimens with cellular morphology typical of either melanoma or benign nevi. Future studies involving a larger number of specimens with borderline morphologies and coupled with long-term patient follow-up will be needed to further evaluate IMP3's value in this regard.

With prognosis and treatment of melanoma relying on sentinel lymph node biopsy findings, accurate histologic analysis of these specimens is vital. When small foci of S-100 or Melan-A positive cells occur within the lymph node trabeculae or parenchyma, diagnosis of metastatic melanoma is problematic. Although the clinical significance of these micrometastases is currently being debated, ${ }^{30}$ it remains the job of the surgical pathologist to identify and diagnose these cells as accurately as possible. This study shows that IMP3 expression has diagnostic utility in distinguishing benign intranodal nevi from metastatic melanoma, and should be considered as a potential tool for the assessment of sentinel lymph node biopsy specimens in patients with melanoma.

\section{Disclosure/conflict of interest}

The authors declare no conflict of interest.

\section{References}

1 Morton DL, Wen DR, Wong JH, et al. Technical details of intraoperative lymphatic mapping for early stage melanoma. Arch Surg 1992;127:392-399.

2 Gershenwald JE, Thompson W, Mansfield PF, et al. Multi-institutional melanoma lymphatic mapping ex- perience: the prognostic value of sentinel lymph node status in 612 stage I or II melanoma patients. J Clin Oncol 1999;17:976-983.

3 Morton DL, Thompson JF, Cochran AJ, et al. Sentinelnode biopsy or nodal observation in melanoma. N Engl J Med 2006;355:1307-1317.

4 Stewart FW, Doan CA. An analysis of the lymphadenopathy question with special reference to Hodgkin's disease tuberculosis. Ann Surg 1931;93:141-152.

5 Ridolfi RL, Rosen PP, Thaler H. Nevus cell aggregates associated with lymph nodes: estimated frequency and clinical significance. Cancer 1977;39:164-171.

6 McCarthy SW, Palmer AA, Bale PM, et al. Nevus cells in lymph nodes. Pathology 1974;6:351-358.

7 Bautista NC, Cohen S, Anders KH. Benign melanocytic nevus cells in axillary lymph nodes. A prospective incidence and immunohistochemical study with literature review. Am J Clin Pathol 1994;102:102-108.

8 Carson KF, Wen DR, Li PX, et al. Nodal nevi and cutaneous melanomas. Am J Surg Pathol 1996;20: 834-840.

9 Murray CA, Leong WL, McCready DR, et al. Histopathological patterns of melanoma metastases in sentinel lymph nodes. J Clin Pathol 2004;57:64-67.

10 Fontaine D, Parkhill W, Greer W, et al. Nevus cells in lymph nodes: an association with congenital cutaneous nevi. Am J Dermatopathol 2002;24:1-5.

11 Holt JB, Sangueza OP, Levine EA, et al. Nodal melanocytic nevi in sentinel lymph nodes. Correlation with melanoma-associated cutaneous nevi. Am J Clin Pathol 2004;121:58-63.

12 Fisher CJ, Hill S, Millis RR. Benign lymph node inclusions mimicking metastatic carcinoma. J Clin Pathol 1994;47:245-247.

13 Hruban RH, Eckert F, Baricevic B. Melanocytes of a melanocytic nevus in a lymph node from a patient with a primary cutaneous melanoma associated with a small congenital nevus. Am J Dermatopathol 1990;12: 402-407.

14 Biddle DA, Evans HL, Kemp BL, et al. Intraparenchymal nevus cell aggregates in lymph nodes: a possible diagnostic pitfall with malignant melanoma and carcinoma. Am J Surg Pathol 2003;27:673-681.

15 Pryor JG, Bourne PA, Yang Q, et al. IMP-3 is a novel progression marker in malignant melanoma. Mod Pathol 2008;21:431-437.

16 Liao $\mathrm{B}, \mathrm{Hu} \mathrm{Y}$, Herrick DJ, et al. The RNA-binding protein IMP-3 is a translational activator of insulin-like growth factor II leader-3 mRNA during proliferation of human K562 leukemia cells. J Biol Chem 2005;280: 18517-18524.

17 Mueller-Pillasch F, Lacher U, Wallrapp C, et al. Cloning of a gene highly overexpressed in cancer coding for a novel KH-domain containing protein. Oncogene 1997;14:2729-2733.

18 Nielsen J, Christiansen J, Lykke-Andersen J, et al. A family of insulin-like growth factor II mRNA-binding proteins represses translation in late development. Mol Cell Biol 1999;19:1262-1270.

19 Hammer NA, Hansen TO, Byskov AG, et al. Expression of IGF-II mRNA-binding proteins (IMPs) in gonads and testicular cancer. Reproduction 2005;130:203-212.

20 Simon R, Bourne PA, Yang Q, et al. Extrapulmonary small cell carcinomas express $\mathrm{K}$ homology domain containing protein overexpressed in cancer, but carcinoid tumors do not. Hum Pathol 2007;38: 1178-1183. 
$21 \mathrm{Gu}$ L, Shigemasa K, Ohama K. Increased expression of IGF II mRNA-binding protein 1 mRNA is associated with an advanced clinical stage and poor prognosis in patients with ovarian cancer. Int J Oncol 2004;24: 671-678.

22 Yantiss RK, Woda BA, Fanger GR, et al. KOC (K homology domain containing protein overexpressed in cancer): a novel molecular marker that distinguishes between benign and malignant lesions of the pancreas. Am J Surg Pathol 2005;29:188-195.

23 Wang T, Fan L, Watanabe Y, et al. L523S, an RNAbinding protein as a potential therapeutic target for lung cancer. Br J Cancer 2003;88:887-894.

$24 \mathrm{Xu} \mathrm{H}$, Bourne PA, Spaulding BO, et al. High-grade neuroendocrine carcinomas of the lung express $\mathrm{K}$ homology domain containing protein overexpressed in cancer but carcinoid tumors do not. Hum Pathol 2007;38:555-563.

25 Lohmann CM, Iversen K, Jungbluth AA, et al. Expression of melanocyte differentiation antigens and ki-67 in nodal nevi and comparison of ki-67 expression with metastatic melanoma. Am J Surg Pathol 2002;26: 1351-1357.

26 Abrahamsen HN, Hamilton-Dutoit SJ, Larsen J, et al. Sentinel lymph nodes in malignant melanoma: extended histopathologic evaluation improves diagnostic precision. Cancer 2004;100:1683-1691.

27 Kucher C, Zhang PJ, Acs G, et al. Can Melan-A replace S-100 and HMB-45 in the evaluation of sentinel lymph nodes from patients with malignant melanoma? Appl Immunohistochem Mol Morphol 2006;14:324-327.

28 Mahmood MN, Lee MW, Linden MD, et al. Diagnostic value of HMB-45 and anti-Melan A staining of sentinel lymph nodes with isolated positive cells. Mod Pathol 2002;15:1288-1293.

29 Zubovits J, Buzney E, Yu L, et al. HMB-45, S-100, NK1/C3, and MART-1 in metastatic melanoma. Hum Pathol 2004;35:217-223.

30 Scolyer RA, Murali R, Satzger I, et al. The detection and significance of melanoma micrometastases in sentinel nodes. Surg Oncol 2008;17:165-174. 